\title{
The Design of Smart Wireless Carbon Dioxide Measuring Instrument Used in Greenhouse
}

\author{
Wengang Zheng ${ }^{1, *}$, Xin Zhang ${ }^{1,2}$, Xiaojun Qiao ${ }^{1}$, Hua Yan $^{2}$, and Wenbiao $\mathrm{Wu}^{2}$ \\ ${ }^{1}$ National Engineering Research Center for Information Technology in Agriculture, \\ 100097, Beijing, China \\ ${ }^{2}$ Beijing Research Center for Intelligent Agricultural Equipment, 100097, Beijing, China \\ \{zhengwg, Zhangx, Qiaoxj, Yanh, Wuwb\} @nercita.org.cn
}

\begin{abstract}
Different crops, seasons, greenhouse's structures and planting area need different concentration of carbon dioxide which fertilized in greenhouse. Measuring the concentration of carbon dioxide accurately in greenhouse is very important to fertilize carbon economically and reasonably Concentration of carbon dioxide measuring equipments were often used in chemical industry and environment fields, these equipments are not suitable for greenhouse's environment and had many disadvantages such as high price, high power consumption and low anti-interference ability. Based on the research of carbon dioxide measuring equipment, this design took part of Non Dispersive Infrared (NDIR) carbon dioxide sensor produced by GSS, embedded Zigbee module provided by Jennic, smart calibration program and solar panel charge system. The results showed that the accuracy was $\pm 30 \mathrm{ppm}$, average power consumption was $2 \mathrm{~mW}$, high consistency, response fast. This design can change the present of carbon dioxide measuring instrument hard to popularize, and make carbon dioxide fertilization accurately.
\end{abstract}

Keywords: greenhouse, carbon dioxide sensor, measuring instruction, Zigbee; low power consumption.

\section{Introduction}

Adding carbon dioxide to fertilize can advance the crop's ratio of photosynthesis and resistance. This technology can also accelerate the growth and improve quality. So it is one of important technologies used in greenhouse production[1][2]. This technology has popularized for twenty years. Many methods have been used for achieving carbon dioxide such as nature ferment, solid carbon dioxide granule, ventilation, chemical reaction etc[3]. The amount of gas mainly calculates by manpower. $\mathrm{CO}_{2}$ detect devices are used rarely for measuring current $\mathrm{CO}_{2}$ concentration. This situation makes fertilize shortage and cannot achieve the desired effect. Once manual calculation or operation errors, higher $\mathrm{CO}_{2}$ concentration can destroy the green plant photosynthetic system. The plant will appear leaf curl and even cause lack of nutrient, high temperature risk of crops[4]. Even more worse, it will do harm to the life of the operator and production human. When the $\mathrm{CO}_{2}$ detecting devices are added for monitoring carbon dioxide concentration in real time,

\footnotetext{
${ }^{*}$ Corresponding author.
} 
it will provided technique supplement for traditional fertilize method and device. And it makes fertilizing carbon dioxide more economical and reasonable.

Parts of agriculture gardens and research institutes can assemble $\mathrm{CO}_{2}$ measuring equipments which are chosen from chemical industry or environment field. U.S.'s ACI, GREYSTONE have explosion-proof encapsulated and high concentration measuring devices which have transform, display, alarm and control functions. Because of the high price, these productions can not widely used in agriculture. Portable $\mathrm{CO}_{2}$ measuring instruments such as Telaire(America)[5], Sense Air (Sweden)[6], Vaisala (Finland) etc. are not suitable for longtime measuring in greenhouse because of the activeness of operator and high power consumption. Domestic low cost $\mathrm{CO}_{2}$ transmitters use semiconductor, solid electrolyte, electrochemistry sensors to measure the $\mathrm{CO}_{2}$ concentration. All these sensor probes have low precision, large drift, high repair ratio and cannot suitable for greenhouse's high temperature and humidity. The domestic research focuses on sensor measurement theory and structure[7]. The fertilize voice generator which promoted crops to absorbing $\mathrm{CO}_{2}$ by audio[8]. But there are few studies of $\mathrm{CO}_{2}$ measuring instruments used in facilities environment. This paper designed a smart wireless carbon dioxide measuring instrument used in greenhouse by using NDIR $\mathrm{CO}_{2}$ sensor and embedded Zigbee module. It had advantages of cost controllable, solar panel powered, low power consumption, high precision self-calibration etc. This instrument could solve the problems, such as hard to be popularized, lack of specific products, low attention.

\section{The Brief Introduction of Carbon Dioxide Measuring Technology}

The mainly used methods for measuring $\mathrm{CO}_{2}$ are titration, chromatography of gases, infra-red spectrum, electrochemistry, semiconductor ceramics, solid electrolyte etc. [9]. The titration method device is complex, low selectivity and sensitivity. Gas chromatography has advantages in sensitivity, accuracy and speed. Greenhouse needs simple measurement instrument with low price, high accuracy and rapid response $\mathrm{CO}_{2}$. But the device based on Gas chromatography method is not suitable for greenhouse's demand for the high price and huge size. So the infra-red, electrochemistry, semiconductor and solid electrolyte sensors are widely used. Electrochemistry and semiconductor sensors have small size, but are not good at accuracy, stability and gas choice. The working life of the electrochemistry sensor is also very short. Table 1 shows the parameters comparison of common $\mathrm{CO}_{2}$ sensors. The solid electrolyte sensor has low price, it is good for measuring on line for a long time. FIGARO(Japan) CDM4160 integrates the sensor of TGS4160[10], which has alarm controlling functions of four concentration points. But its' long warm-up time and high power consumption features are not suitable for accurate measuring in greenhouse. The NDIR sensor has advantages in accuracy and stability. GE/Telaire (America) 6004 which already been stopped producing has large market share before. Module 6113 is replaced. Korea's ELT H500 has low cost and small size. It is suitable for the testing production which does not sensitive with the power. Although domestic $\mathrm{CO}_{2}$ sensor MG811's price is very low, it is not good for greenhouse application environment because its' accuracy interfered by humidity. By 
comprehensive comparison, U.K. GSS Company takes solid state light module and reference diode to make sensor lower price, faster, lower power, higher accuracy and long time stability. Low power module C20LP/C100LP with 10mW consumption will be brought at recent time. It is fit for combining with wireless sensor networks. This design uses this module to build greenhouse smart wireless carbon dioxide measuring instrument.

Table 1. Parameters comparison of common $\mathrm{CO}_{2}$ sensors or module

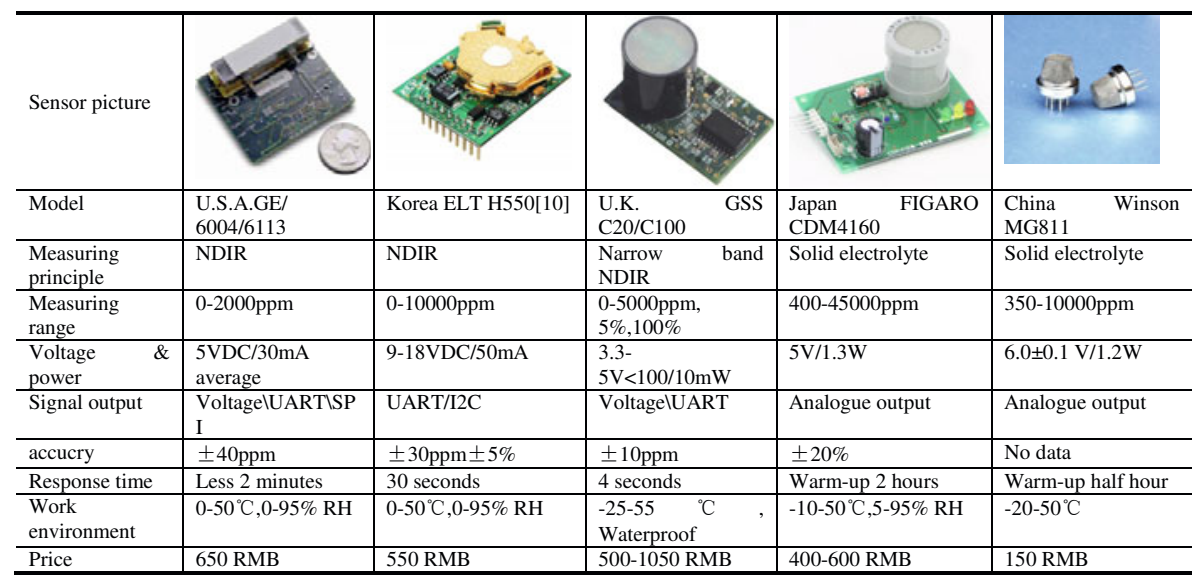

\section{The Structure of Smart Wireless $\mathrm{CO}_{2}$ Measuring Equipment}

$\mathrm{CO}_{2}$ concentration measuring instrument in greenhouse has some functions below. (1) Transmitter function that could provide $4-20 \mathrm{Ma}, 0-5 \mathrm{~V}$, or frequency output, it is convenience to link with the collection and controlling devices. (2) Function of record and display. (3) Long distance transmitting function. Multi-point measurement and long distance transmission by wireless or wired to form network. (4) Controlling and alarm function, It could control the actuator and alarm devices in greenhouse by comparing data gathered with setting alarm points or critical point. All these functions were used to achieve the precise the concentration of $\mathrm{CO}_{2}$ fertilization [12].

This design takes wireless sensor networks technology for measuring $\mathrm{CO}_{2}$ concentration by functional module method. The sensor is installed in radiation shield. This instrument can gather, transform, display, storage the data. It also can communicate with PC or data collector by wireless of Zigbee. All the functions of this design could add or delete to control the price. And this can optimize with the performance, price and power consumption. Multi-points or single point monitor in one greenhouse, star network and point to point network are used by PC, Co-ordinator and end device. (Figure 1). Chain network was used for multi-points monitor in greenhouse. (Figure 2). This paper introduces the design of $\mathrm{CO} 2$ measuring instrument. 


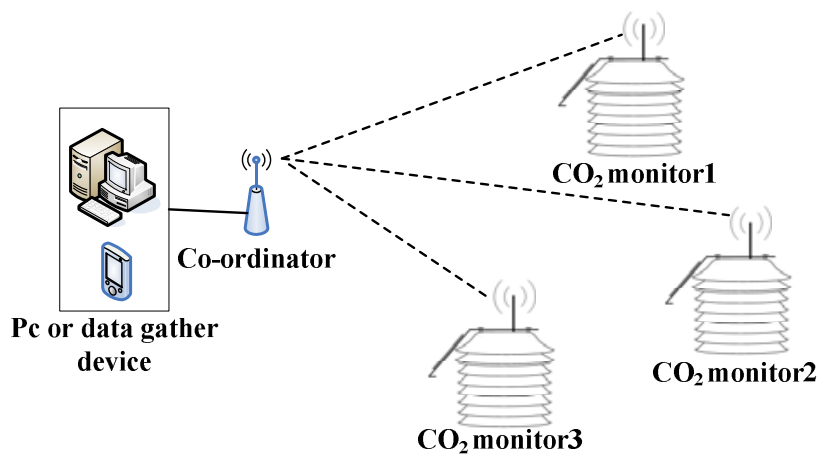

Fig. 1. Multi-points or single point measurement in single greenhouse

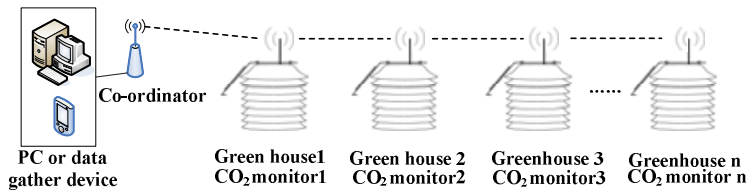

Fig. 2. Multi-points monitor in greenhouses

\section{The Hardware Design of the Equipment}

The design has to consider the whole functions. But it can reduce the function module to lower the cost base on the real application. $3 \mathrm{~V}$ voltage is used in this system. All chips' power supply is managed. 2A lithium battery and solar panel were power for system's longtime working.

This instrument's hardware structure is showed in Figure 3. The Jennic company's third of generation Zigbee module JN5148 supports Zigbee Pro protocol It has low power consumption (low power module receive and transmit current is $17.5 / 15 \mathrm{~mA}$, high power module receive and transmit current is $23 / 110 \mathrm{~mA}$ ), abundance resource on chip (32 bit MCU, 128Kb RAM, 128kB ROM, 12bit ADC/DAC, two UARTs etc.). The functions of this device can come true by embedding application routine.

GSS's CO2 sensor is linked with the JN5148 by UART port. It can transfer the data gathered, calibrate and setting instruction. $\mathrm{CO}_{2}$ module's calibrate and zeroing ports were linked with the switched and MCU's I/O port which could make calibration and zeroing to come true by manual operation and automation .The chip of $74 \mathrm{HC} 04$ achieves the electrical level transform because $\mathrm{CO}_{2}$ module needs $5 \mathrm{~V}$ voltage supply.

The segment LCD display module is used to display the gathered data. FM31256 integrates real time clock, watch dog timer and FRAM in a chip[14]. It provides accuracy real time clock and $256 \mathrm{Kbit}$ storage space. This IC communicated with I2C port.

Transmitter module transfers digital data to 4-20mA output by IC AD694. This chip links with the module's 12 bit DAC converter, and transfers $0-2 \mathrm{~V}$ which DAC output into standard current signal. 


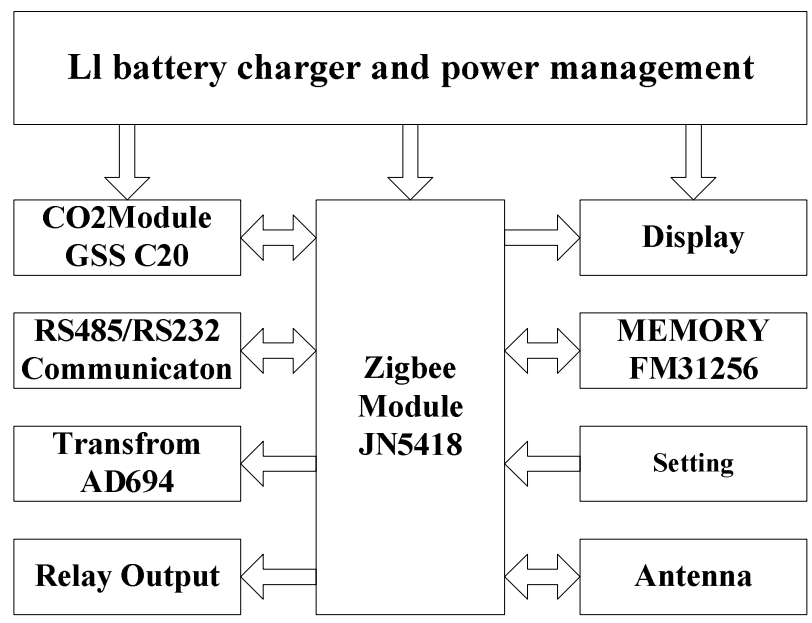

Fig. 3. Hardware design structure

This system provides dry contact by relay module. This dry contact could link with the motor and alarm devices to achieve controlling and alarm functions.

RS232/RS485 module occupies one of the URAT port. The module is used for network communication and program processing. This design provides switches for setting number, communication channel, baud rate etc.

$1-2 \mathrm{~W}$ solar panel is used for charging the $2 \mathrm{Ah}$ lithium battery. The charger IC CN3082 could finish this function. CN3082 can adjust charge current base on solar panel's current output ability[16]. The part of power management uses NCP500 to provide $3.3 \mathrm{~V}$ voltage for system. Power switch IC TPS2044 controls the power supply of the lcd, communication IC. This power management method achieves minimum power consumption.

\section{The Software Design of the Equipment}

The software design base on Zigbee chip JN5148 uses the ZPS Configuration Editor and JenOS Configuration Editor which are integrated in IDE Eclipse for head file. Added with application program and linked to finish the development of software[17].

\subsection{Overall Thinking of Software Design}

The software is made up of message event which comes from ZPS protocol, Task and time trigged application program. All these message, task and software timer application are driven by the JenOS, and communicates with the ZPS protocol. Basic end device net parameters are set by ZPS Configuration. As showed in Figure 4, system has six message events. The App_msgRelaycontrol, App_msgTransmitter, 
App_msgPowermanage, App_msgCalibrate are used for making the controlling, transmit, low power and calibrate functions to come true. App_msgSensorevent is used to storage, display, communication etc. App_msgZpsevent was used for management App_taskSensornode.

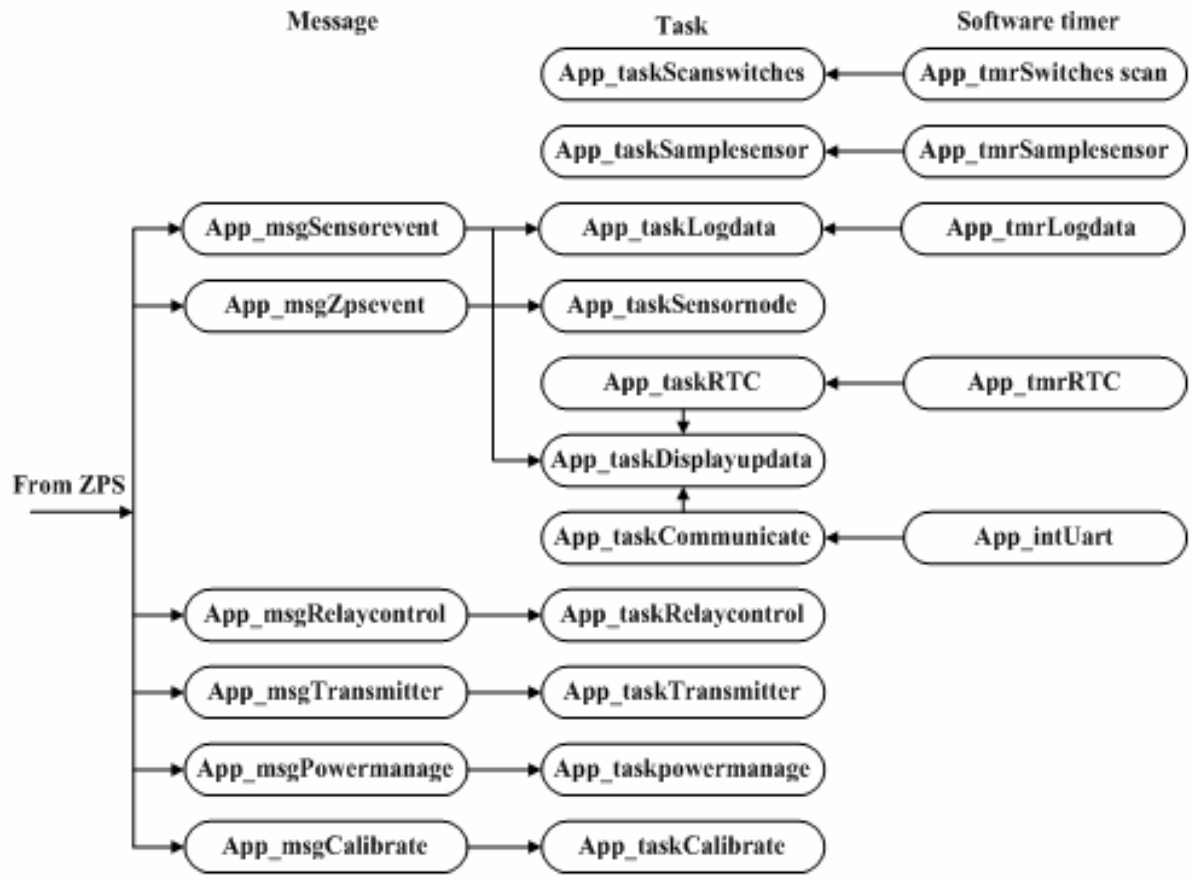

Fig. 4. Software structure

\subsection{Communication with $\mathrm{CO}_{2}$ Module}

JN548 communication with $\mathrm{CO}_{2}$ module by setting and calling UART1 API which included in Integrated Peripherals API. Related communication settings function is shown as follows:

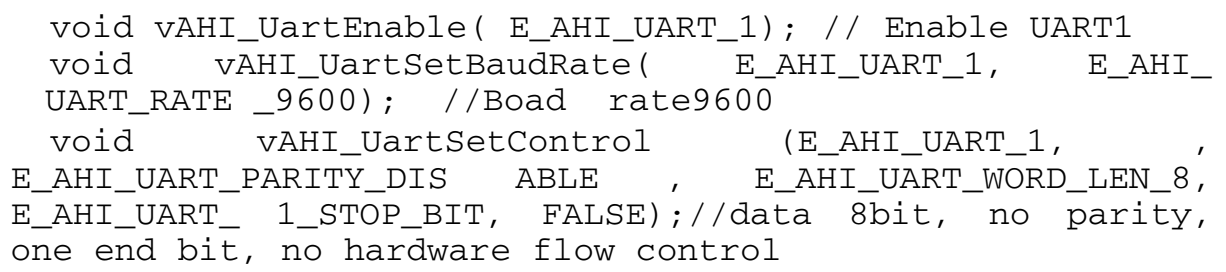

Through setting the FIFO, interrupt of UART and recall function which used as application routine to achieve the software calibration, fast response, filter, LED's signal intension, temperature of PCB etc. functions. 
Taking instruction: M6\r\n Output: Z00057z00060 for example, it means that fast output is $570 \mathrm{ppm}$, and filter output is $600 \mathrm{ppm}$. Other function achieved will not be listed here one by one.

\subsection{The Calibration Function}

Although the $\mathrm{CO}_{2}$ module has been rigorously calibrated before delivery, measuring data will not accurate like before for long time using. So it is necessary to calibrate the sensor. Module can use the nitrogen gas or other gas which does not include $\mathrm{CO}_{2}$ to calibrate the sensor by pulling down the pin2 of sensor. The surrounding environment is also used for calibration by pulling down the ninth pin of sensor.

For compensating the problem of easy to interference when using the air calibration method. Over sample algorithm is used in the software design[18]. This algorithm takes 12 hours as acquisition cycle in outdoor no interference air. The data measured each 15 minutes are compared with the reference data(380-400ppm) to get average calibrate factor. Then the factor is used for compensating the $\mathrm{CO}_{2}$ concentration. Because the implementation is simple, this paper will not list the specific algorithm program.

\section{Experiment and Testing}

This wireless $\mathrm{CO}_{2}$ measuring instrument has already tested and calibrated in laboratory and application site. Wireless communication, communication distance, real time and bit error rate etc. Jennic has the test reports of module and antenna. Its' features meet the demands of the greenhouse -site wireless network applications.

Different standard concentration $\mathrm{CO}_{2}$ gas which provided by National Standard Material Research Center. The instrument's small size and wireless communication ways are suitable for calibrating in the sample gas bar. The data are showed in Table 2.

Device $1^{*}$ is the instrument used in greenhouse for ten months. Device 1 was calibrated by the over sample algorithm based on Device $1 . \mathrm{CO}_{2}$ sensor should calibrate when used over one year. As Table 2 shows the accuracy is lower than $30 \mathrm{ppm}$. From the data of device 2 and 3, we can see that the sensor's accuracy could meet the demands of greenhouse production's acquirement.

Table 2. Calibrate and test table of $\mathrm{CO}_{2}$ sensors (Unit: ppm)

\begin{tabular}{l|ccccccccccc}
\hline Standard gas & 0 & 200 & 400 & 600 & 800 & 1000 & 1200 & 1400 & 1600 & 1800 & 2000 \\
\hline Device 1* & 0 & 180 & 390 & 570 & 870 & 980 & 1170 & 1360 & 1570 & 1760 & 1950 \\
Device 1 & 0 & 200 & 410 & 610 & 800 & 990 & 1210 & 1420 & 1610 & 1820 & 1970 \\
Device 2 & 0 & 190 & 400 & 600 & 810 & 1010 & 1220 & 1410 & 1620 & 1810 & 2000 \\
Device 3 & 0 & 200 & 390 & 600 & 780 & 980 & 1200 & 1390 & 1560 & 1780 & 1990
\end{tabular}

In Beijing Daxin district's Caiyu mum base, two instruments were used in number one greenhouse. The data of Jan.5th 2010 were show in Figure 5. From figure 5, it is to see that $\mathrm{CO} 2$ fertilization is needed after nine o' clock. Its consistency can meet the measure's requirement . 


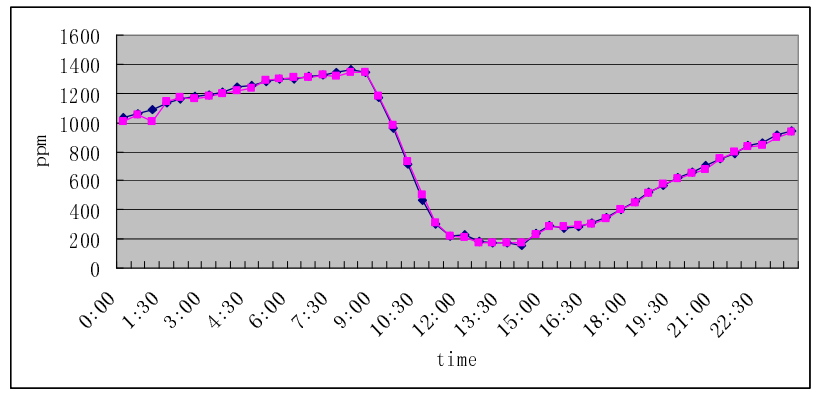

Fig. 5. Data in greenhouse

\section{Conclusions}

This paper aims at $\mathrm{CO}_{2}$ fertilization which lacks of suitable facilities $\mathrm{CO}_{2}$ detection device and depends on imports and industrial equipment to integrate the Zigbee embedded technology, low power $\mathrm{CO}_{2}$ sensor、over sampling calibrate program (accuracy is $<= \pm 30 \mathrm{ppm}$ ) and efficient solar power system for designing a kind of smart wireless carbon dioxide measuring instrument. This instrument has a lot of advantages, such as low power consumption (average power is $2 \mathrm{~mW}$ ), wireless communication, controlling and alarm and cost controllable (basic function is 500RMB) etc. Wireless Zigbee Pro protocol is used in wireless sensor network which provides a new model of $\mathrm{CO}_{2}$ measurement. This design provides equipment to greenhouse $\mathrm{CO}_{2}$ fertilization, and achieves the precise control the concentration of $\mathrm{CO}_{2}$ fertilization.

Acknowledgments. It is a project supported by national supported project "The design and application on integration of management \& control of facilities agriculture technology"(2008BAD6B00), national 863 project "The key and integration technology of wireless measuring and controlling networks." (2006AA10Z253), and Beijing science and technology program topic "The design of special sensors and acquisition of agricultural equipment” (D101105046310002).

\section{References}

1. Chen, Y., Li, M., Jang, J.: The carbon dioxide fertilization technology in facilities vegetables. West North Horticulture 1, 43 (2009)

2. Shan, X.: The carbon dioxide fertilization in greenhouse. Anhui agriculture aviso 12(13), 149 (2006)

3. Wang, T.: The application and countermeasure of greenhouse $\mathrm{CO}_{2}$ fertilization technology. The science of Shanxi Agruiculture 34(2), 49-51 (2006)

4. Liu, $\mathrm{H} .:$ The essential technology of $\mathrm{CO}_{2}$ fertilization in greenhouse. Agruiculture science and devices 176(2), 77-78 (2008)

5. $\mathrm{CO}_{2}$ OEM module-6004, http: / /www . tongdy. com 
6. SenseAir, Model Esense Carbon Dioxide sensor and transmitter (2003), http: / /www. senseair.com

7. Yuan, C., He, B., Han, X., et al.: Research advance in detection of carbon dioxide. Acta Agriculture Jiangxi 21(6), 133-136 (2009)

8. Hou, T., Li, B., Teng, G., et al.: The application of plant audio controlling technology in facility vegetable production 21(6), 133-136 (2009)

9. He, R.: Design and experiment research of carbon dioxide detection based on infrared spectrum absorption theory. Jinlin University (2009)

10. Figaro: Product information of CDM4146-Pre- calibrated module for carbon dioxide Rev:12/07

11. GSS Ltd, The datasheet of C20E Carbon Dioxide Sensor, Rev CE 05/09

12. Ma, S., Ma, Y., Zhang, L., et al.: The designing of Carbon Dioxide Density Detection Instrument Used in Agriculture. Agricultural Research (12), 104-115 (2007)

13. Jennic Data sheet- JN5148-001-Myy IEEE802.15.4/ Zigbee PRO Module Family, $1 v 1(2009)$

14. RAMTRON. The datasheet of FM31256, http: / /www . ramtron. com. cn

15. Analog Device, The datasheet of 4-20mA transmitter AD694, REV.B (2002), http: / / www . analog.com

16. CONSONANCE, The data sheet of CN3082. REV 1.0, http: / / www. consonance-elec.com

17. Jennic Zigbee PRO Configuration Guide, JN-UG-3065v 1.1, 2009.12

18. TELAIRE, 6000 series module $\mathrm{CO}_{2}$ sensor UART and SPI communications protocols, v01 